\title{
磑化铟(InI)多晶的水平区熔提纯
}

\author{
徐朝鹏，张䂞，王倩，纪亮亮，李亚可 \\ (燕山大学 信息科学与工程学院, 河北省特种光纤与光纤传感重点实验室, 秦皇岛 066004)
}

摘 要: 采用水平区熔法对由两温区气相输运法制备的 InI 多晶进行提纯，探索高纯、单相 InI 多晶的制备工艺。 通过 $\mathrm{X}$ 射线粉末衍射仪、扫描电子显微镜以及电感耦合等离子体原子发射光谱仪对水平区熔提纯前后的 InI 多 晶的晶格结构、形貌组分以及杂质浓度进行了表征。结果表明，经过水平区熔提纯后的 InI 多晶晶格结构完整，质 量较高, 晶格参数为 $a=0.476 \mathrm{~nm}, b=1.278 \mathrm{~nm}, c=0.491 \mathrm{~nm}$, 与理论值十分接近; In、I 化学配比得到了有效调节, 其化学配比接近理论化学配比 1: 1; 中间产物含量及杂质浓度显著降低。以提纯后 InI 多晶为原料, 用提拉法生 长出的 InI 单晶电阻率达到 $10^{10} \Omega \cdot \mathrm{cm}$ 。

关 键 词: 碘化铟; 多晶合成; 气相输运; 区域熔融; 提纯

中图分类号: 078 文献标识码: A

\section{Horizontal Zone Refining of Indium Iodide (InI) Polycrystal}

\author{
XU Zhao-Peng, ZHANG Lei, WANG Qian, JI Liang-Liang, LI Ya-Ke
}

(School of Information Science and Engineering, The Key Laboratory for Special Fiber and Fiber Sensor of Hebei Province, Yanshan University, Qinhuangdao 066004, China)

\begin{abstract}
The indium iodide (InI) polycrystal which was synthesized by two-zone vapor transporting method was purified by using horizontal zone refining method. Preparation process on high quality and single phase InI polycrystal was successfully fixed. The lattice structure, morphology, component and impuritily concentration of InI crystal were investigated by X-Ray Diffraction (XRD), Scanning Electron Microscope-Energy Dispersive Spectrometer (SEM-EDS) and Inductively Coupled Plasma Atomic Emission Spectrometry (ICP-AES). The results indicate that the InI polycrystal after refinement has better lattice structure and higher quality than before. The lattice constants of $a, b$ and $c$ are $0.476 \mathrm{~nm}, 1.278 \mathrm{~nm}$ and $0.491 \mathrm{~nm}$, respectively, which are very close to the theoretical values. The stoichiometric ratio of In to I is effectively modified and close to the theoretical stoichiometric ratio 1:1. The intermediate products and impurities concentrations decrease significantly. An InI single crystal with high resistivity (about $10^{10} \Omega \cdot \mathrm{cm}$ ) is grown by Czochralski (CZ) method with the purified InI polycrystal obtained in this work.
\end{abstract}

Key words: indium iodide; polycrystalline synthesis; vapor transporting; zone refining; purification

碘化铟(InI)是一种极有应用前景的室温核辐射 探测器材料 ${ }^{[1]}$, 属于III-VII型化合物, 具有层状结构
的底心正交晶体。InI 晶体的高原子序数 $\left(Z_{\mathrm{In}}: 49, Z_{\mathrm{I}}\right.$ : $53)$ 和高密度 $\left(5.31 \mathrm{~g} / \mathrm{cm}^{3}\right)$ 特性使 InI 探测器对伽马射

收稿日期: 2015-03-11; 收到修改稿日期：2015-05-21

基金项目: 河北省应用基础研究计划重点基础研究项目(13961103D); 中国电子科技集团公司第四十六研究所创新基金 (CJ20120208); 河北省高层次人才资助项目(C2013003040); 燕山大学青年教师自主研究计划(13LGA011) Key Basic Research Program of Applied Basic Research Project of Hebei (13961103D); Innovation Fund of China Electronic Technology Group Corporation NO.46 Research Institute (CJ20120208); High Level Talent Funded Project of Hebei (C2013003040); Young Teachers Independent Research Project of Yanshan University (13LGA011)

作者简介: 徐朝鹏(1977-), 男, 博士研究生, 教授. E-mail: xuzhaopeng1@163.com 
线具有与 $\mathrm{CdTe}$ 相当的阻止能力, 保证了探测器具 有较高的探测效率。 InI 晶体的禁带宽度为 $2.0 \mathrm{eV}$, 可以有效抑制载流子的热激发, 从而在室温下获得 低噪声性能。 $\mathrm{InI}$ 晶体还具有电阻率高 $\left(\sim 10^{11} \Omega \cdot \mathrm{cm}\right)$ 和载流子迁移率-寿命积大 $\left(\sim 7.2 \times 10^{-5} \mathrm{~cm}^{2} / \mathrm{V}\right)$ 等特 点。与 $\mathrm{HgI}_{2} 、 \mathrm{CdTe} 、 \mathrm{CdZnTe}(\mathrm{CZT}) 、 \mathrm{GaAs}$ 等探测 器材料相比, 制备 InI 晶体的原料 In 和 I 的毒性也 相对要小 ${ }^{[2-3]}$ 。

国外对 InI 晶体已经开展了一定研究, 如 1956 年, Peretti 等 ${ }^{[4]}$ 就完成了 In-I 系统相图的绘制。1979 年, Nakamura 等 ${ }^{[5]}$ 利用气相法在玻璃安鶕中成功生 长出 InI 晶体。之后很长一段时间, 由于提纯技术以 及生长技术的制约, InI 晶体的研究没有取得大的进 展。1993 年, Kolinko 等 ${ }^{[6]}$ 对 InI 的能带结构进行了 计算, 证实 InI 的晶体结构是类斜方晶系 $\alpha$-型 TII 结 构, 而且具有 TII 类似的态分布。2011 年, 美国伊 利诺伊理工大学的 Nicoara 等 ${ }^{[7]}$ 用两温区气相输运 法合成了 InI 多晶, 并利用 Czochralski 法生长了 InI 晶体, 但其电阻率不高, 只有 $10^{9} \Omega \cdot \mathrm{cm}$ 。根据近几年 的研究, InI 多晶的合成技术受多种因素控制, 容易 导致合成出的多晶杂质浓度高、化学配比偏离等现 象。为了满足 InI 半导体器件的要求, InI 单晶中杂 质浓度需要严格控制, 电活性杂质典型的浓度值要 求 $<1.0 \times 10^{-5}$, 而高纯、单一相的 InI 多晶原料是生长 高质量 InI 单晶的必备条件。因此, 需要对合成的 InI 多晶进行提纯, 从而调节多晶的化学配比, 降低其 杂质浓度。

InI 晶体在熔点 $\left(365^{\circ} \mathrm{C}\right)$ 至室温间不存在固-固相 变, 因此用熔体法就可以较容易地生长出 $\mathrm{InI}$ 晶体 ${ }^{[8]}$ 。 本工作以高纯 $\mathrm{I}_{2}(5 \mathrm{~N})$ 和 $\operatorname{In}(7 \mathrm{~N})$ 为原料合成出 $\operatorname{InI}$ 多晶, 使用区熔法对多晶进行提纯，分析了提纯前后 $\mathrm{InI}$ 多晶的化学配比和杂质浓度。并以提纯后 InI 多晶 为原料生长出高电阻率的 InI 单晶。

\section{1 实验方法}

\section{1 多晶合成装置}

在可抽真空的两温区水平管式合成炉中进行 InI 的多晶合成。高温区和低温区由两套独立的电阻 线圈分别调控。测温系统由热电偶和温控仪组成。 合成炉和温场曲线示意图如图 1 所示。

\subsection{InI 多晶的制备}

In-I 化合物的相图 ${ }^{[4]}$ 如图 2 所示。由相图可知, 在制备 InI 多晶过程中会产生 $\mathrm{InI}_{2} 、 \mathrm{InI}_{3} 、 \mathrm{InI}$ 三种直 接产物, 其中 $\mathrm{InI}_{2}$ 易分解。当 $\mathrm{I}$ 的原子百分比在 $0 \sim$

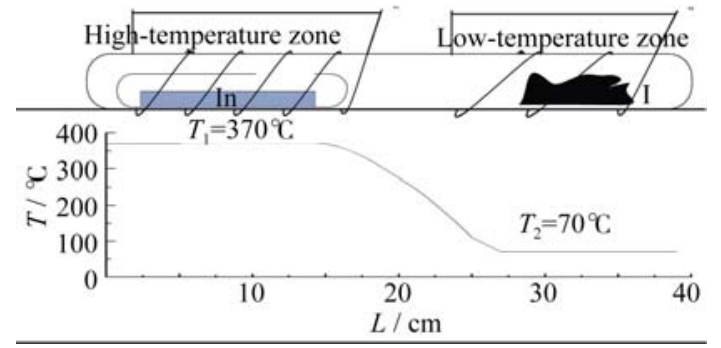

图 1 InI 多晶合成炉和温场曲线示意图

Fig. 1 Schematic diagram of synthesis furnace and temperature curve for InI polycrystal

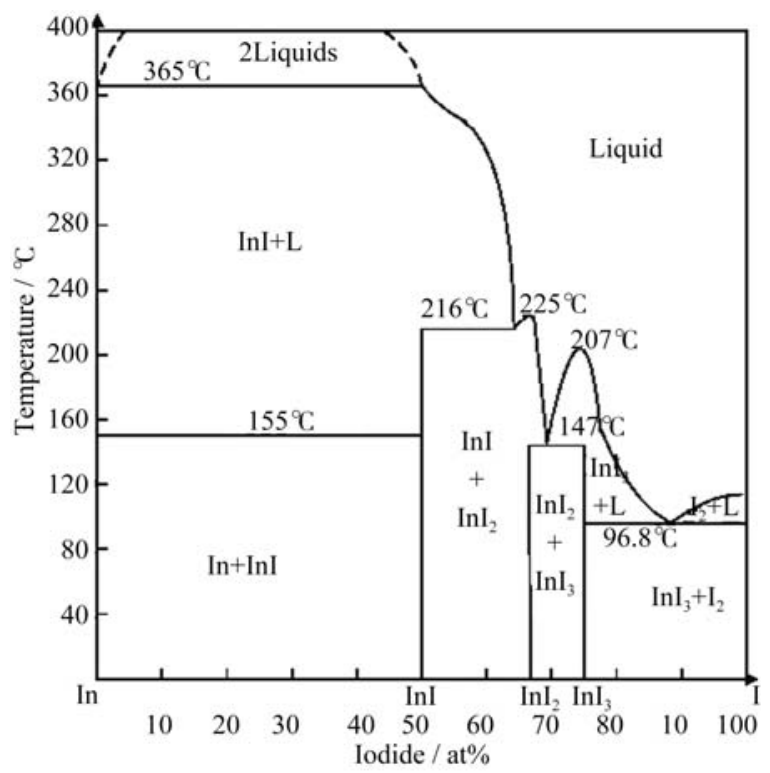

图 2 In-I 化合物相图 ${ }^{[4]}$

Fig. 2 Indium-Iodine compound phase diagram ${ }^{[4]}$

$50 \mathrm{at} \%$ 之间，温度在 $365{ }^{\circ} \mathrm{C}$ 以上时，相图上展示为一 个 InI+In 的液态共熔成分。根据相图，我们就可以 确定 InI 多晶的合成工艺。

采用高纯 $I_{2}(5 N)$ 和 $I n(7 N)$ 为原料, 按化学配比 并适量富 In 进行配料。通过两温区气相输运法合成 InI 多晶, 配料时适当富 In 可确保合成的原料尽可 能地符合理想化学配比。单质 In 的熔点比 InI 低, 密 度比 $\mathrm{InI}$ 大，熔融的 $\mathrm{InI}$ 和 In 具有分层现象 ${ }^{[9]}$ 。因此, 富余的 In 能够在合成反应结束后，沉积于石英安瓿 的底部，与合成的 InI 多晶分离 ${ }^{[10]}$ 。

装料前, 石英安瓿与石英舟先用甲醇溶液再用 丙酮溶液分别冲洗 3 4 次, 最后用去离子水冲洗干 净, 放到干燥箱中烘干备用。装料时, 先称取 $19.4 \mathrm{~g}$ In 单质放入右端开口的石英舟中，再将石英舟放入 安瓿中; 然后称取 $20 \mathrm{~g} \mathrm{I}$ 单质装入已经放有石英舟 的安瓿另一端。对石英安瓿抽真空，当真空度达到 $2.0 \mathrm{~Pa}$ 后，向安瓿中通入氩气，将安鶕中的空气排 出, 此过程反复进行 5 8 次, 确保真空度达到 $2.0 \mathrm{~Pa}$ 
以下后进行封结。将封装好的合成安瓿放入两温区 合成炉内, 安瓿有小舟的一端置于高温区, 另一端 置于低温区, 低温区不加热, 高温区合成升温曲线 如图 3 所示。如图 3 所示, 高温区升温至 $100^{\circ} \mathrm{C}$ 保温 $1 \mathrm{~h}$ 左右, 使高温区的微量 $\mathrm{I}$ 蒸汽在低温区凝结。根 据相图, 当温度低于 $216^{\circ} \mathrm{C}$ 时, 会生成黄色的 $\mathrm{InI}_{3}$, 因此从 $100^{\circ} \mathrm{C}$ 升温到 $380^{\circ} \mathrm{C}$ (高于 $\mathrm{InI}$ 的熔点 $365^{\circ} \mathrm{C}$ ) 应该尽量快。在 $380^{\circ} \mathrm{C}$ 保温 $3 \mathrm{~h}$, 使 In 完全融化, 与 $\mathrm{I}$ 蒸汽在气-液界面处充分反应生成 $\mathrm{InI}{ }^{[11]}$ 。将高温反 应区的温度升高到 $450^{\circ} \mathrm{C}$ 并保温 $3 \mathrm{~h}$, 使 $\mathrm{InI}$ 多晶与 少量残留的熔融态 In 发生热质交换, 进一步与 I 蒸 汽发生反应。反应结束后将高温区缓慢降到室温。

实验中合成的 InI 多晶如图 4 所示, 呈紫红色块 状物，具有金属光泽。石英舟底部附着的银白色金 属是未反应完全剩余的 In。剩余的 In 和合成出的 InI 多晶间有明显的分层现象, 将银白色 In 去除, 即得 到 InI 多晶。

\section{3 提纯原理}

区域熔融提纯法被广泛用来提纯半导体材料 及其他晶体材料 ${ }^{[12]}$ 。它利用了晶体材料凝固时的分 凝(偏析)现象, 即凝固过程中出现的凝固部分的杂 质浓度和溶液中杂质浓度不同的现象, 将杂质集 中到凝固部分的前部和后部, 而其中间部分获得 了提纯。

分凝系数 $K_{0}=C_{\mathrm{S}} / C_{\mathrm{L}}$ 在很大程度上影响着材料

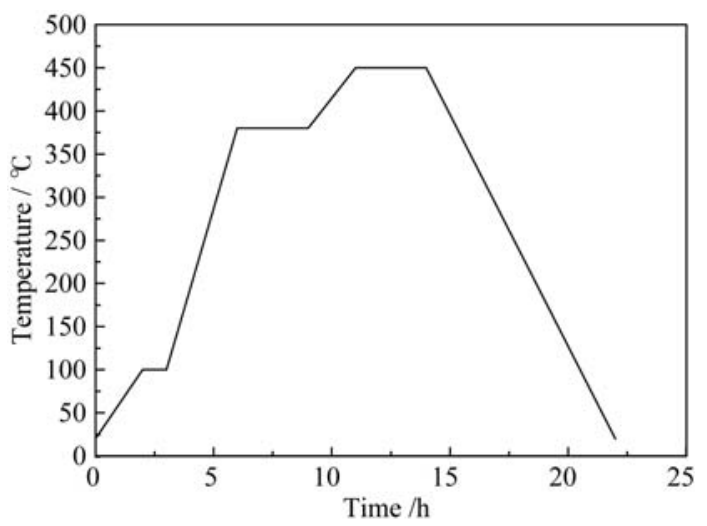

图 $3 \mathrm{InI}$ 多晶合成升温曲线图

Fig. 3 Temperature curve for InI polycrystal

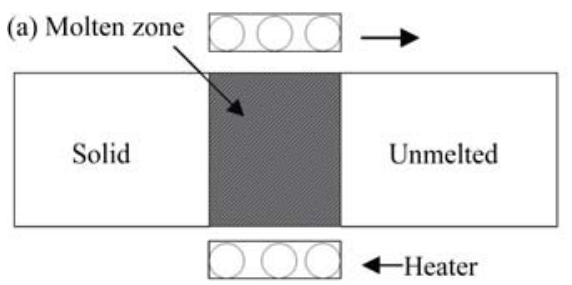

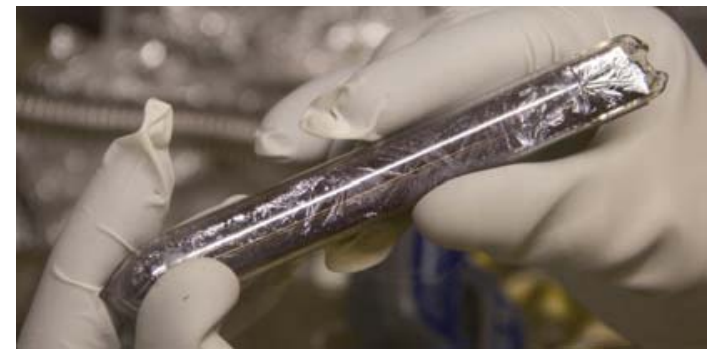

图 4 合成的 InI 多晶

Fig. 4 Synthesized InI polycrystal

的提纯效果, 其中 $C_{\mathrm{S}}$ 为处于平衡状态时固相中杂质 的浓度, $C_{\mathrm{L}}$ 为液相中杂质的浓度。对于 $K_{0}<1$ 的杂质, 液相中的杂质浓度大于固相中的杂质浓度, 杂质向 多晶右端富集; 而 $K_{0}>1$ 的杂质，杂质则向多晶的左 端富集。InI 多晶中的大部分杂质如 $\mathrm{Fe} 、 \mathrm{~B} 、 \mathrm{Ni}$ 、 $\mathrm{Mg} 、 \mathrm{Al}$ 等, 分凝系数远小于 1 , 都会富集到多晶右 端。InI 多晶中部分杂质的分凝系数如表 1 所示。

在同等条件下，区熔次数对提纯效果也有很大 的影响。随着区熔次数的增加, 杂质含量会逐渐减 少, 但在经过一定次数的区熔后, 杂质的分布达到 极限, 此时再增加次数也不会提高提纯效果。根据 文献[13]的研究，区熔 20 次后杂质将达到极限分布， 此时再增加区熔次数不仅对杂质浓度影响不大，反 而可能导致杂质倒流，影响提纯效果。因此本实验 采用了 20 次区熔的提纯工艺。

\section{4 多晶提纯装置}

在可抽真空的水平管式提纯炉中进行 InI 多晶 的提纯。步进电机带动电阻线圈对 InI 多晶进行提 纯。区熔提纯过程及 InI 多晶提纯炉示意图如图 5 所示。

\subsection{InI 多晶提纯}

采用与前述多晶合成相同的装料清洗工艺。把

表 1 单质碘和单质铟所含的部分杂质及其分凝系数 Table 1 Segregation coefficient of elements in iodine and indium

\begin{tabular}{cccccc}
\hline Element & $\mathrm{Mg}$ & $\mathrm{Ni}$ & $\mathrm{Ca}$ & $\mathrm{Cr}$ & $\mathrm{Fe}$ \\
\hline$K_{0}$ & 0.013 & 0.163 & 0.071 & 0.018 & 0.028 \\
\hline
\end{tabular}

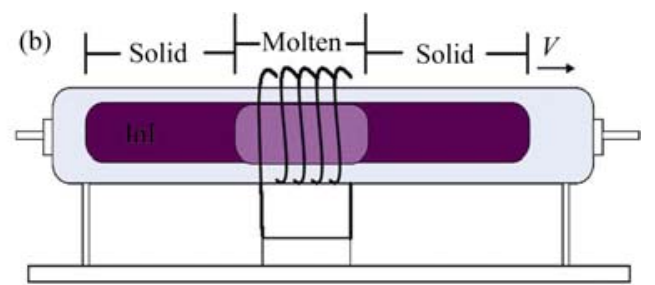

图 5 区熔提纯过程(a)及提纯炉(b)示意图

Fig. 5 Schematic diagram of zone refining process (a) and zone refining furnace (b) 
合成的 InI 多晶分成小块, 取高质量的 InI 多晶块进 行封装, 将封装好的石英安瓿放入区熔提纯炉中。 加热圈以 $370{ }^{\circ} \mathrm{C}$ 的温度, $2 \mathrm{~cm} / \mathrm{h}^{[3]}$ 的速度从石英安瓿 左侧开始对 InI 多晶进行提纯。到达右端后, 再以 $2 \mathrm{~cm} / \mathrm{s}$ 的速度快速回到左端, 以防止杂质倒流, 影 响提纯效果。此时一次区熔过程结束, 开始进行下 一次区熔。重复上述过程 20 次, 以保证杂质浓度到 达分布极限。

\section{2 结果与讨论}

\section{$2.1 X$ 射线粉末衍射分析}

从提纯前后的 InI 多晶样品中各取少量试样, 在玛瑙研钵中研磨成粉末状, 采用 D/max-2500 型 X 射线粉末衍射仪对样品的晶型结构进行分析。测试 条件: $\mathrm{Cu}$ 靶 $\mathrm{K} \alpha$ 线, 工作电压为 $40 \mathrm{kV}$, 工作电流为 $100 \mathrm{~mA}$, 入射波长为 $0.15406 \mathrm{~nm}$, 扫描范围 $(2 \theta)$ $20^{\circ} \sim 70^{\circ}$ 。

图 6 是提纯前后 $\mathrm{InI}$ 多晶的 XRD 图谱。从图 6 可以看出, 两个测试样品的衍射图谱与 InI(PDF\#790534)标准衍射图谱均基本一致, 峰形尖锐。但是图 6(a)中提纯前 InI 多晶的图谱在 $25^{\circ} 、 36^{\circ} 、 57^{\circ}$ 附近 有几个明显的杂相峰, 经过比对与 $\mathrm{InI}_{2}(\mathrm{PDF} \# 35-$ 1446)峰形一致, 说明提纯前 InI 多晶中含有杂相 $\mathrm{InI}_{2}$ 。通过 Jade 软件对 XRD 图谱进行分析, 得到 提纯前后 InI 的晶格参数差别不大, 基本一致, 提 纯后 InI 晶格参数为: $a=0.476 \mathrm{~nm}, b=1.278 \mathrm{~nm}$, $c=0.491 \mathrm{~nm} ; \alpha=\beta=\gamma=90^{\circ}$, 结果与 Jones 和 Templeton ${ }^{[14]}$ 计算的结果: $a=0.475 \mathrm{~nm}, b=1.276 \mathrm{~nm}, c=0.491 \mathrm{~nm}$; $\alpha=\beta=\gamma=90^{\circ}$ 非常接近, 说明提纯后样品为高纯、单一 相的 InI 多晶。

\subsection{SEM 形貌观察和 EDS 成分分析}

使用蔡司 SUPRA 55 扫描电子显微镜(SEM)观 察提纯前后 InI 多晶解理面的组织形貌, 并用能谱 仪(EDS)分析断面成分。提纯前后 SEM 形貌如图 7 所示, 解理断面的元素组分分析如表 2 所示。

由图 7 可以看出, 提纯前后 $\mathrm{InI}$ 多晶解理断面变 化不大, 都呈现明显的片层状结构。由表 2 可以看 出, 提纯前晶体组成元素原子比 $I n: I \approx 1: 1.12$, 提纯 后 In: $I \approx 1: 0.97$ 。合成实验采用富 In 配比, 但提纯前 多晶富 I, 且化学配比偏离严重, 造成这种结果的原 因是多方面的: 其一, In 密度大于 InI, 熔融的 InI 多
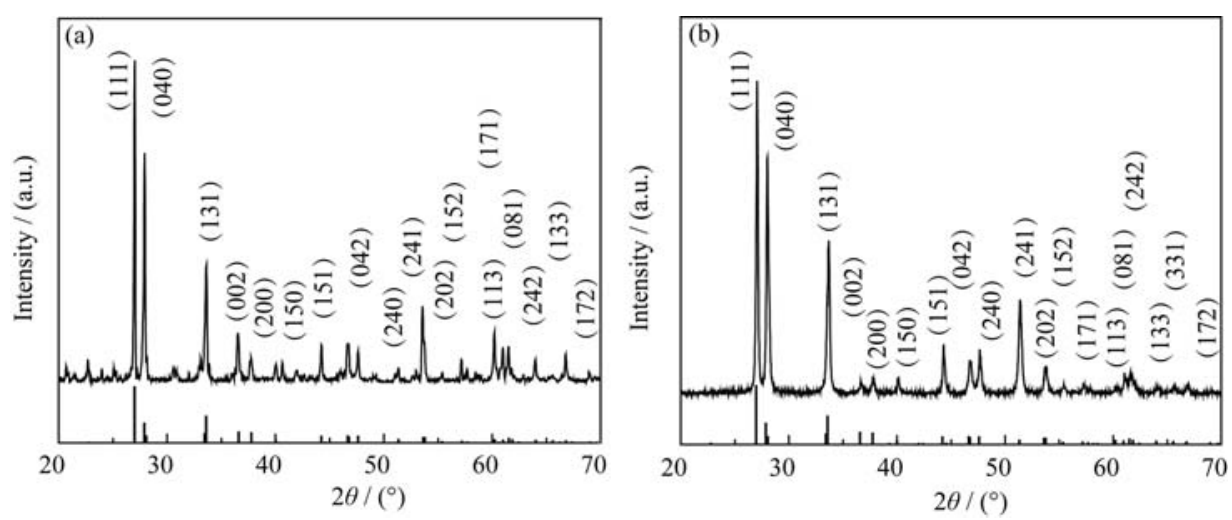

图 6 提纯前后 InI 多晶样品的 XRD 图谱

Fig. 6 XRD patterns of InI polycrystalline sample before (a) and after (b) refinement
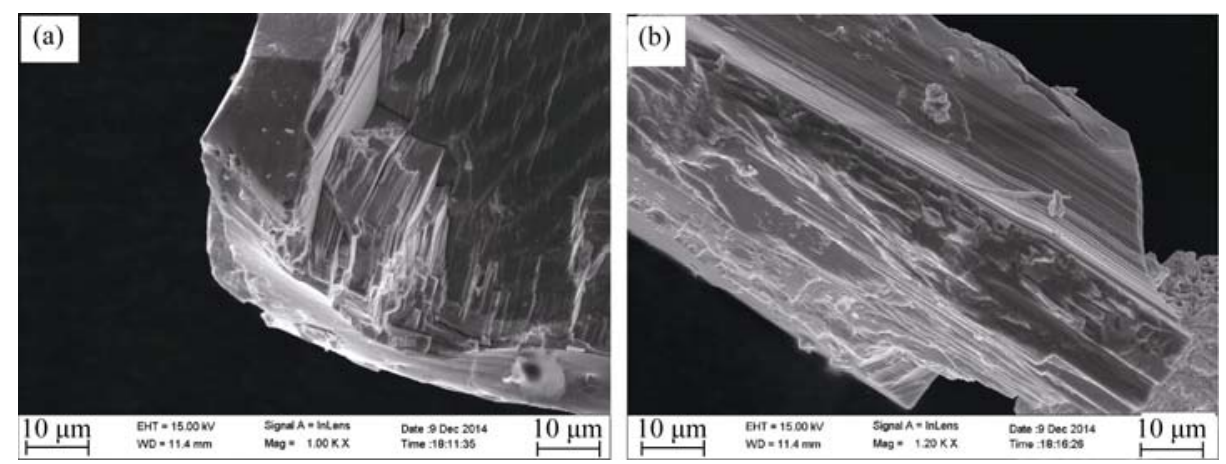

图 7 提纯前(a)后(b)InI 多晶的 SEM 照片

Fig. 7 SEM images of InI polycrystal before (a) and after (b) refinement 
表 2 提纯前后 InI 多晶样品的 EDS 分析结果

Table 2 EDS analysis results of InI polycrystalline samples before and after refinement

\begin{tabular}{cccc}
\hline Sample & In $/$ at $\%$ & I/at $\%$ & Total $/$ at $\%$ \\
\hline Before refinement & 47.13 & 52.87 & 100 \\
After refinement & 50.69 & 49.31 & 100 \\
\hline
\end{tabular}

晶会浮于未反应的少量 In 上面，阻碍反应的进行, 因此有部分 I 剩余并冷凝在 InI 多晶表面; 其二, 受限于合成工艺，反应过程中有中间产物 $\mathrm{InI}_{2}$ 、 $\mathrm{InI}_{3}$ 生成。提纯后多晶富 In, 化学配比偏离程度明 显降低，与理想化学配比非常接近。这主要是因 为区域熔融法在处理 InI 多晶时，未完全反应的 I 会与 In 进一步反应生成 InI，同时 InI 多晶中的中 间产物, $\mathrm{InI}_{2}$ 受热易分解, $\mathrm{InI}_{3}$ 在加热情况下会汽化 并向安瓿的右端富集，冷凝在安瓿表面，与多晶 分离。

提纯后的 InI 晶体的 In、I 原子比接近 1: 1, 说 明区域熔融法对 InI 多晶 In、I 化学配比的调节效果 明显。

\section{3 电感耦合等离子体原子发射光谱分析}

使用电感耦合等离子体原子发射光谱仪(ICPAES)对 InI 多晶进一步进行组分分析，确定提纯前 后多晶中杂质元素的种类和含量, 以及提纯对 In、I 化学配比的影响。

从提纯前后的 $\mathrm{InI}$ 多晶样品中间部分各取 $400 \mathrm{mg}$ 试样，使用 $98 \%$ 浓盐酸配制测试所需的分析试液。
使用 iCAP 6300 Radial 型 ICP-AES 分析仪对样品分 析试液中杂质元素进行定性、定量分析。该型仪器 的元素检出限针对不同元素有一定差别，一般能达 到 $0.00 X \mu \mathrm{g} / \mathrm{mL}$ 级别。ICP-AES 测试结果如表 3 所 示。由表 3 可以看出, 提纯之后, $\mathrm{Li}$ 和 $\mathrm{Cr}$ 两种元素 杂质浓度已低于检出限, 其他杂质浓度都显著降低, 说明区域熔融法对于 I 和 In 中常见的分凝系数 $K_{0}<$ 1 的杂质提纯效果比较显著。且提纯后 In、I 化学配 比得到了明显调节，与能谱仪(EDS)分析结果基本 一致。

\subsection{InI 单晶电阻率测试}

实验采用提拉法 $(\mathrm{CZ})$ 生长 $\mathrm{InI}$ 单晶。在生长前 首先采用分子泵对生长炉室抽真空, 真空度降至 $0.1 \mathrm{~Pa}$ 时充氩气，以排出其中的空气。重复 5 8 次 后调小氩气流量进行升温, 生长温度 $360{ }^{\circ} \mathrm{C}$, 提拉 速度 $3 \mathrm{~cm} / \mathrm{h}$, 旋转速度 $20 \mathrm{r} / \mathrm{min}$ 。生长出的 $\mathrm{InI}$ 单晶 呈紫红色，尺寸为 $\phi 20 \mathrm{~mm} \times 40 \mathrm{~mm}$, 如图 8 所示。

将 InI 单晶沿生长方向切割成晶片, 经机械抛 光后，采用 $0.5 \% \mathrm{Br}-\mathrm{MeOH}$ 腐蚀液对其表面进行化 学抛光处理, 处理后样品尺寸为 $\phi 20 \mathrm{~mm} \times 0.5 \mathrm{~mm}$ 。 采用四探针电阻测试系统对 $\mathrm{InI}$ 的电阻率进行测量, 该系统通过采集晶片表面电压，然后对采集到的电 压取平均值计算该点的电阻率。本次实验采集了晶 片中间约 20 个点, 结果如图 9 所示。根据欧姆定律, 计算出 $\mathrm{InI}$ 单晶的电阻率约为 $3.8 \times 10^{10} \Omega \cdot \mathrm{cm}$, 比文 献[7]报道值高一个量级。

表 3 提纯前后 InI 多晶样品的各元素浓度

Table 3 Concentrations of elements in InI polycrystalline before and after refinement

\begin{tabular}{ccccccccccc}
\hline Elements & $\mathrm{In} / \mathrm{at} \%$ & $\mathrm{I} / \mathrm{at} \%$ & $\begin{array}{c}\mathrm{Li} / \\
\left(\mu \mathrm{g} \cdot \mathrm{mL}^{-1}\right)\end{array}$ & $\begin{array}{c}\mathrm{Cr} / \\
\left(\mu \mathrm{g} \cdot \mathrm{mL}^{-1}\right)\end{array}$ & $\begin{array}{c}\mathrm{Cu} / \\
\left(\mu \mathrm{g} \cdot \mathrm{mL}^{-1}\right)\end{array}$ & $\begin{array}{c}\mathrm{Mg} / \\
\left(\mu \mathrm{g} \cdot \mathrm{mL}^{-1}\right)\end{array}$ & $\begin{array}{c}\mathrm{Al} / \\
\left(\mu \mathrm{g} \cdot \mathrm{mL}^{-1}\right)\end{array}$ & $\begin{array}{c}\mathrm{Zn} / \\
\left(\mu \mathrm{g} \cdot \mathrm{mL}^{-1}\right)\end{array}$ & $\begin{array}{c}\mathrm{Fe} / \\
\left(\mu \mathrm{g} \cdot \mathrm{mL}^{-1}\right)\end{array}$ & $\begin{array}{c}\mathrm{Ni} / \\
\left(\mu \mathrm{g} \cdot \mathrm{mL}^{-1}\right)\end{array}$ \\
\hline Before refinement & 47.58 & 52.41 & 0.0504 & 0.0469 & 0.0790 & 0.1287 & 0.1256 & 0.2885 & 0.6436 & 0.8187 \\
After refinement & 50.74 & 49.25 & -- & -- & 0.0093 & 0.0107 & 0.0311 & 0.0291 & 0.0565 & 0.0740 \\
\hline
\end{tabular}

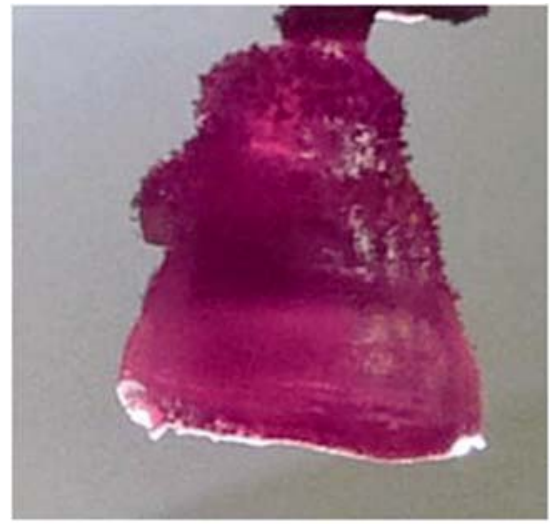

图 8 提拉法生长的 InI 单晶

Fig. 8 InI crystal grown by $\mathrm{CZ}$ method

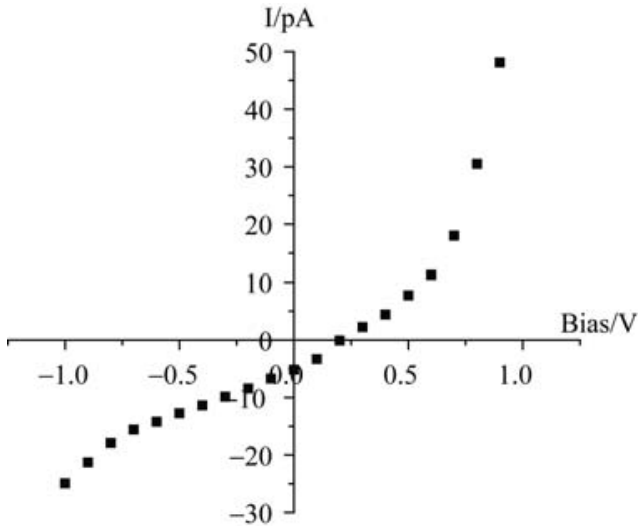

图 9 InI 单晶的 $I-V$ 曲线

Fig. 9 Current-voltage curve of InI crystal 


\section{3 结论}

本工作在传统两温区气相输运法合成的 $\mathrm{InI}$ 多 晶基础上，采用区域熔融法对 InI 多晶进行提纯，探 索出一整套高质量室温核辐射探测材料 InI 多晶的制 备工艺。测试结果表明，提纯后 InI 多晶的纯度得到 显著提高, In 和 I 的化学配比更加接近理论化学配比, 用其生长的 InI 单晶电阻率能达到 $3.8 \times 10^{10} \Omega \cdot \mathrm{cm}$ 。

\section{参考文献:}

[1] BISWAS K, DU M H. First principles study of native defects in InI. J. Appl. Phys., 2011, 109(11): 113518.

[2] ONODERA T, HITOMI K, SHOJI T. Fabrication of indium iodide X-and gamma-ray detectors. IEEE Tran. Nucl. Sci., 2006, 53(5): 3055-3059.

[3] BHATTACHARYA P, GROZA M, CUI Y, et al. Growth of InI single crystals for nuclear detection applications. J. Cryst. Growth, 2010, 312(8): 1228-1232.

[4] PERETTI E A. Thermal analysis of the indium-iodine system. $J$. Am. Chem. Soc., 1956, 78(22): 5745-5746.

[5] NAKAMURA K, OHNO N, FUJITA M, et al. Photoluminescence of orthorhombic InI. J. Lumin., 1979, 18(1): 381-384.

[6] KOLINKO M I. Electronic energy-structure calculations for orthorhombic InI single crystals. J. Phys. Condens. Mat., 1994, 6(1): 183-202.

[7] NICOARA I, NICOARA D, BERTORELLO C, et al. Czochralski Growth of Indium Iodide and Other Wide Bandgap Semiconductor Compounds. MRS Proceedings. Cambridge University Press, 2011, 1341(1): 95-104.

[8] SQUILLANTE M R, ZHOU C, ZHANG J, et al. InI nuclear radiation detectors. IEEE Tran. Nucl. Sci., 1992, 40(4): 364-366.

[9] HAYNES W M. CRC Handbook of Chemistry and Physics. CRC Press, 2013: 4-18, 6-129.

[10] ZHAO XIN, JIN YING-RONG, HE YI, et al. Modified two-zone vapor transporting method for $\mathrm{PbI}_{2}$ polycrystal synthesis. Chinese $J$. Inorg. Chem., 2011, 27(2): 298-302.

[11] 介万奇. 晶体生长原理与技术. 北京：科学出版社, 2010: 179-188.

[12] LANG SHU-LING, ZHANG LI, CHEN SONG, et al. Preparation technique of high purity cobalt. Rare Metals, 2010, 34(6): 911-918.

[13] LI WEN-LIANG, LUO YUAN-HUI. Preparation of high purity zinc by zone refining. Rare Metals, 2011, 35(4): 537-542.

[14] JONES R E, TEMPLETON D H. The crystal structure of indium (I) iodide. Acta Crys., 1955, 8(12): 847. 